\title{
A NOTE ON MEASURABILITY AND ALMOST CONTINUITY
}

\author{
MAXIM R. BURKE AND DAVID H. FREMLIN
}

(Communicated by R. Daniel Mauldin)

\begin{abstract}
We prove that it is consistent with ZFC that there exist a measurable function $f:[0,1] \rightarrow \omega_{1}$ which is not almost continuous.
\end{abstract}

DEFINITION. If $Y$ is a topological space, a function $f:[0,1] \rightarrow Y$ is measurable if $f^{-1}(G)$ is Lebesgue measurable for each open $G \subset Y$, and is almost continuous if for every $\varepsilon>0$, there is a measurable set $E$ with $\mu E>1-\varepsilon$, and $\left.f\right|_{E}$ continuous.

In $[2$, Theorem 2B], Fremlin proves

THEOREM 1. If $X$ is a Radon measure space and $Y$ is a metric space, then a function $f: X \rightarrow Y$ is measurable iff it is almost continuous.

(See [2] for the definition of a Radon measure space. All we need to know here is that $[0,1]$ with Lebesgue measure is such a space.)

Assuming GCH there is the following result for nonmetrizable spaces $Y$.

THEOREM 2 [2, THEOREM 3G]. (GCH) If $X$ is Radon and $\varsigma$ is an ordinal (with the order topology), then every function $f: X \rightarrow \varsigma$ is measurable iff it is almost continuous.

What happens to Theorem 2 if GCH is dropped is left open in [2] even when $X=[0,1], \varsigma=\omega_{1}$. In this paper we show that Theorem 2 can fail in this case:

THEOREM 3. If $\mathfrak{c}=2^{\aleph_{0}}=2^{\aleph_{1}}$ and there is a collection $\left\{N_{\xi}: \xi>\mathfrak{c}\right\}$ of null (= Lebesgue negligible) subsets of $[0,1]$ such that $\forall L \in[\mathfrak{c}]^{\omega_{1}}, \bigcup_{\xi \in L} N_{\xi}=[0,1]$, then there is a function $f:[0,1] \rightarrow \omega_{1}$ which is measurable but not almost continuous.

REMARK. The hypothesis of Theorem 3 holds in the model obtained by adding $2^{\aleph_{1}}$ Cohen reals to a model of ZFC. (The collection $\left\{N_{\xi}: \xi<\mathfrak{c}\right\}$ is obtained by taking the translates of a fixed null comeager set $H$ by the elements of a Luzin set of size $c$. See [1].)

PROOF OF THEOREM 3. Let $\left\langle C_{\xi}: \xi<\mathfrak{c}\right\rangle$ enumerate the club subsets of $\omega_{1}$. We define a collection $\left\{E_{\eta}: \eta<\omega_{1}\right\}$ of null sets satisfying

(i) $\forall \eta<\omega_{1}\left(E_{\eta} \subset N_{\eta}\right)$,

(ii) $\forall \xi<\mathfrak{c}\left(\bigcup_{\eta \in C_{\xi}} E_{\eta} \supset[0,1] \backslash N_{\xi}\right)$,

(iii) $\forall \eta, \eta^{\prime}<\omega_{1},\left(\eta \neq \eta^{\prime} \Rightarrow E_{\eta} \cap E_{\eta^{\prime}}=\varnothing\right)$.

Given $x \in[0,1]$, let $A(x)=\left\{\xi<\mathfrak{c}: x \notin N_{\xi}\right\} \cup\{0\}$. This is a nonvoid countable set. Let $\eta(x)$ be the least member of $\bigcap_{\xi \in \mathcal{A}(x)} C_{\xi}$ s.t. $x \in N_{\eta(x)}$. Define $E_{\eta}=\{x \in$ $[0,1]: \eta=\eta(x)\}$. It is easy to check (i), (ii), and (iii).

Received by the editors November 19, 1986.

1980 Mathematics Subject Classification (1985 Revision). Primary 28A05.

(C) 1988 American Mathematical Society $0002-9939 / 88 \$ 1.00+\$ .25$ per page 
Now define $f:[0,1] \rightarrow \omega_{1}$ by $f^{-1}(\{\eta\})=E_{\eta}$. By (ii), $f^{-1}(C)$ is measurable when $C$ is closed, and thus $f$ is measurable. But $f$ is not almost continuous since if $K$ is compact, $\mu K>0$, and $\left.f\right|_{K}$ is continuous then $f(K)$ is a compact subset of $\omega_{1}$; thus $f(K)$ is countable and so $K$ is a countable union of null sets, contradicting $\mu K>0$.

This completes the proof.

REMARK. J. Cichon has pointed out that the function $f$ can be constructed so that $f^{-1}(A)$ is null iff $A$ is nonstationary: Let $\left\{B_{\gamma}: \gamma<\mathfrak{c}\right\}$ be a decomposition of $[0,1]$ into disjoint Bernstein sets, let $\left\langle S_{\gamma}: \gamma<\mathfrak{c}\right\rangle$ enumerate the stationary subsets of $\omega_{1}$. Then in the proof above choose $\eta(x)$ from $\bigcap_{\xi \in A(x)} C_{\xi} \cap S_{\gamma}$ where $\gamma$ is chosen so that $x \in B_{\gamma}$.

\section{REFERENCES}

1. K. Kunen, Random and Cohen reals, Handbook of Set-Theoretic Topology, North-Holland, 1984.

2. D. H. Fremlin, Measurable functions and almost-continuous functions, Manuscripta Math. 33 (1981), 387-405.

Department of Mathematics, University of Toronto, Toronto, Ontario, M5S 1A1, CANADA

Department of Mathematics, University of Essex, Colchester CO4 3SQ, ENGLAND 